\title{
Photometric Properties of the Hubble Space Telescope Fine Guidance Sensors
}

\author{
K. Zwintz, R. Kuschnig, W. W. Weiss, A. Witeschnik \\ Institute for Astronomy, University of Vienna, Türkenschanzstrasse 17, \\ A-1180 Vienna, Austria
}

\begin{abstract}
To reach lower noise levels with the Hubble Space Telescope Fine Guidance Sensors (FGS) the photometric properties as well as the systematic effects affecting the data have been investigated.
\end{abstract}

\section{Deadtime, Color Term, Sensitivity, and Systematic Effects}

The FGS datasets which show no intrinsic variability, and for which more or less consistent magnitudes and colors are available, were used for a new determination of deadtime, color term and sensitivity. The deadtimes for FGS1 and FGS3 are 50 ns; for FGS2 the value is 100 ns. (see Weiss et al., 1999)

With the data obtained during the Hubble Deep Field program (1995 December) the following systematic effects have been investigated: 1) The South Atlantic Anomaly (SAA) is a distortion of the geomagnetic field of the Earth. No observations are performed during passages of the HST through this zone. But already at the borders of the zone the instruments measured increased intensities. Datapoints influenced by the SAA have to be excluded from further analysis (Zwintz et al. 1999). 2) The harmonic variation which is modulated by the orbital period of the HST of 96 min can be explained as stray light coming from the Earth. To model this effect the phase angle between the HST and the Sun with respect to the Earth has been calculated with simple spherical trigonometry (Zwintz et al. 1999). 3) During an 11-h time interval the count rates of both PMTs in the FGS Y-Axis dropped by about $20 \%$. The electronics recovered towards the end of the $11-\mathrm{h}$ period to the count rate observed before the interval. The origin of this effect is presently unknown (Zwintz et al. 1999).

Acknowledgments. This project received funding from the Österreichische Nationalbank (project 8014) and the Fonds zur Förderung der Wissenschaftlichen Forschung (project S-7303 AST).

\section{References}

Weiss, W. W., Zwintz, K., Kuschnig, R., \& Witeschnik, A. 1999, Comm. Ast. No. 129

Zwintz, K., Kuschnig R., Weiss W. W., Gray, R. O., \& Jenkner, H. 1999, A\&A, 343, 899 